\title{
Molecular Complexity of Certain Homologous Series of Condensed Benzenoid Hydrocarbons: Limiting Values of the Patency Index and the Index of Spanning-Tree Density
}

\author{
Roger B. Mallion, ${ }^{1, *}$ Paul Pollak, ${ }^{2}$ Paweł J. Skrzyński ${ }^{3}$
}

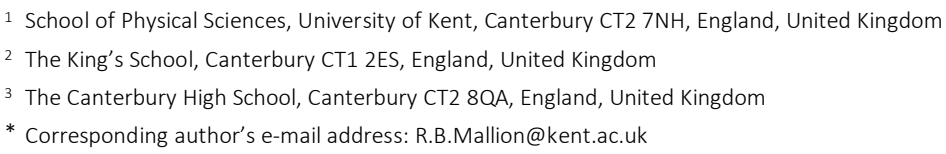

RECEIVED: August 30, 2017 \ REVISED: November 16, 2017 ネ ACCEPTED: November 16, 2017

\begin{abstract}
Two indices of molecular complexity, the Patency Index (2017) and the Spanning-Tree Density (2003), are applied to three homologous series of condensed benzenoid hydrocarbons. Calculation of the Spanning-Tree Density requires finding the number of spanning trees in a given molecular graph, which may be achieved by applying the Cycle Theorem (2004) or, in the case of planar graphs in a planar embedding, the theorem of Gutman, Mallion \& Essam (1983). To compute the Patency Index, it is necessary to count the number of ladders in the embedded molecular graph. This is done by means of the Dual Cycle Theorem (2017). In the latter, a ladder is conceived of as the edge-set relating to faces as the edge-set of a spanning tree relates to vertices. For a planar graph in a planar embedding, the number of ladders is equal to the number of spanning trees. The three homologous series investigated here are the Linear $[n]$-Acenes $\left(A_{n}\right)$, the $[n]$-Phenacenes $\left(P h_{n}\right)$ and the $[n]$-Helicenes $\left(H_{n}\right)$ (the latter of which are geometrically non-planar but graph-theoretically planar). For these three series, the Spanning-Tree Densities and the Patency Indices may be obtained in closed form and so their behaviour as $n \rightarrow \infty$ is easily examined. Though neither index distinguishes between individual members of the three series $\left(A_{n}, P h_{n}\right.$ and $\left.H_{n}\right)$ for a specified value of $n$, this does not preclude the possibility that, within each series, either index may exhibit correlation with physical or chemical data.
\end{abstract}

Keywords: molecular complexity, spanning trees, ladders of embedded graphs, patency index, spanning-tree density.

\section{INTRODUCTION}

$\mathbf{H}$ OW INTRICATE[1] a molecular structure is has, in recent years, frequently been called the complexity of the molecule; for reviews, see, for example, Ref. [1] (and the Refs. [1-7,11-13,19,22,24-26] that are cited in Ref. [1]), Ref. [2] (and Refs. [11-24] cited there) and see also Refs. [3-6] of the present paper. This appellation is perhaps unfortunate because much of this work involves the application of Graph Theory and the term 'complexity' was already long used by graph-theorists (e.g., Ref. [7]) to denote the number of spanning trees in a graph. (Recall that a spanning tree - necessarily of a one-component graph $G$ - is a connected acyclic sub-graph that contains all the vertices of $G^{[8]}$.) Deliberately avoiding the word 'complexity' - and introducing instead the word 'intricacy' - Trinajstić and one of the present authors (RBM) defined ${ }^{[1]}$ what they called the Spanning-Tree Density of a molecular graph $(S T D(G))$ as the ratio

$$
\operatorname{STD}(G)=\frac{t(G)}{{ }^{e} C_{(v-1)}},
$$

where $t(G)$ is the number of spanning trees in the molecular graph $(G)$, which is taken to have $e$ edges and $v$ vertices. ${ }^{(e} C_{(v-1)}$, often also written as

$$
\left(\begin{array}{c}
e \\
v-1
\end{array}\right)
$$


is the number of ways in which $(v-1)$ distinct objects can be selected from $e$ unlike objects.) STD $G$ ) thus effectively represents the probability that, if precisely $(v-1)$ edges are removed at random from the graph, that process results in the creation of a spanning tree of the graph. $t(G)$ may be computed in many ways (for reviews see, for example, Refs. [9-14]) but the present authors ${ }^{[2]}$ have frequently applied what Kirby et al. called the Cycle Theorem ${ }^{[15,16]}$ for suitably embedded general one-component molecular-graphs or the theorem of Gutman, Mallion \& Essam ${ }^{[17]}$ for the special case of planar graphs in a planar embedding. In an attempt to relate $\operatorname{STD}(G)$ for a molecular graph to the 'complexity' of that molecule, Mallion \& Trinajstić ${ }^{[1]}$ considered the reciprocal of the Spanning-Tree Density and postulated that the greater the 'Reciprocal Spanning-tree Density' of a given molecular graph, the more intricate (in the sense of being 'complicated') that molecular structure should be considered to be.

Very recently, Kirby and the present authors have offered (in this journal) ${ }^{[2]}$ a Dual of the Cycle Theorem, for a graph embedded on a closed surface, in which the entities counted are not spanning trees ${ }^{[8]}$ but, rather, what might be regarded as 'duals' of the edge-sets of spanning-trees. These we define in Ref. [2] as ladders. An index for a molecular graph $G$, called by Kirby et al. ${ }^{[2]}$ the Patency Index $(P(G))$ of $G$, was there defined as follows:

$$
P(G)=\frac{/(G)}{{ }^{e} C_{(f-1)}},
$$

where $I(G)$ is the number of ladders ${ }^{[2]}$ in $G$, which has $e$ edges, $v$ vertices and $f$ faces. The method of calculating $/(G)$ is described in Ref. [2]. It was shown there ${ }^{[2]}$ that, for a planar graph on a 'planar' embedding (i.e., one not using generic circuits, defined in Ref. [15], even if the surface is capable of sustaining such),

$$
P(G)=\operatorname{STD}(G) \text {. }
$$

In this note we apply these two indices to some control sets of structures which constitute well-known homologous series of condensed benzenoid hydrocarbons ${ }^{[18,19]}$ - in the first instance, we consider the Linear [ $\left.n\right]$ Acenes - and we show that, for these series, it is possible to solve for $P(G)$ and $S T D(G)$ in closed form and we are, thereby, able to examine the limiting behaviour of these two indices as $n \rightarrow \infty$. We label the molecular graphs of this first series of conjugated systems, the Linear Acenes, as $A_{n}$ (with $A_{1}$ standing for Benzene, $A_{2}$ for Naphthalene, etc.) Figure 1 shows $A_{4}$ (Tetracene) embedded on the surface of a sphere (denoted by the red circle - please see Ref. [2] for details of the implications of this notation.) Consideration is then extended to two analogous homologous series (the [n]-Phenacenes and the [ $n$ ]-Helicenes - illustrated later),

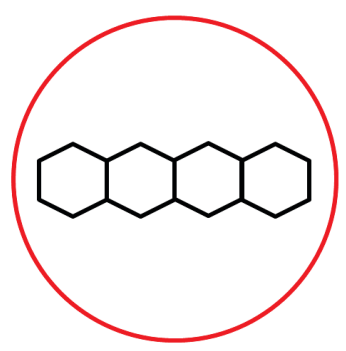

Figure 1. $A_{n}$ embedded on the surface of a sphere (with, here, $n=4)$. The red circle has the significance that was explained in Ref. [2] - effectively, it makes this representation of the sphere topologically faithful.

as well as to some more-compact tessellations of $n$ hexagons that constitute the molecular graphs of other condensed, benzenoid hydrocarbons.

\section{ALGEBRAICAL ARGUMENTS TO OBTAIN EXPRESSIONS FOR $S T D\left(A_{n}\right)$ AND $P\left(A_{n}\right)$ IN CLOSED FORM}

The sub-determinant within the face-to-face incidence-matrix $^{[2,20]}$ for $A_{4}$ (say, $\left.f(4)\right)$, obtained by retaining only the entries relating to the hexagonal faces - or, alternatively, the corresponding determinant obtained by an application of the theorem of Gutman et al. ${ }^{[17]}$ — is

$$
f(4)=\operatorname{det}\left(\begin{array}{cccc}
6 & -1 & 0 & 0 \\
-1 & 6 & -1 & 0 \\
0 & -1 & 6 & -1 \\
0 & 0 & -1 & 6
\end{array}\right)=1189
$$

and equals the number of spanning trees (and also the number of ladders ${ }^{[2]}$ ) in $A_{4}$. It is not difficult to see that

$$
f(n)=6 f(n-1)-f(n-2),
$$

and also that $f(1)=6$ and $f(2)=35$. The recurrence relation (4), with these initial conditions, can be solved in the usual way to give

$$
f(n)=\frac{(3+\sqrt{8})^{n+1}}{\sqrt{32}}-\frac{(3-\sqrt{8})^{n+1}}{\sqrt{32}}
$$

\section{APPROXIMATIONS AND NUMERICAL CALCULATIONS}

As $f(n)$ is an integer the expression in equation (5) can be replaced by

$$
f(n)=\text { the integral part of } \frac{(3+\sqrt{8})^{n+1}}{\sqrt{32}},
$$


i.e.,

$$
f(n)=\left[\frac{(3+\sqrt{8})^{n+1}}{\sqrt{32}}\right] .
$$

As a check on this we may note that

$$
f(3)=\operatorname{det}\left(\begin{array}{ccc}
6 & -1 & 0 \\
-1 & 6 & -1 \\
0 & -1 & 6
\end{array}\right)=204
$$

and, using equation (6), we find that

$f(3)=$ the integral part of $\frac{(3+\sqrt{8})^{4}}{\sqrt{32}}=[204.00015]=204$.

It is noteworthy that, even for a small value of $n$, the decimal part of

$$
\frac{(3+\sqrt{8})^{n+1}}{\sqrt{32}}
$$

is very small. If the Patency Index for $A_{n}$ is evaluated from

Table 1. Approximate values of $P\left(A_{n}\right)$ (to 4 decimal places), calculated from equation (7), for Linear Acenes $A_{1}-A_{20}$

\begin{tabular}{lc}
\hline$n$ & $P\left(\mathrm{~A}_{n}\right)$ (to 4 decimal places) \\
\hline 1 & 1.0000 \\
2 & 0.6364 \\
3 & 0.3643 \\
4 & 0.1987 \\
5 & 0.1054 \\
6 & 0.0549 \\
7 & 0.0282 \\
8 & 0.0144 \\
9 & 0.0073 \\
10 & 0.0036 \\
11 & 0.0018 \\
12 & 0.0009 \\
13 & 0.0005 \\
14 & 0.0002 \\
15 & 0.0001 \\
16 & 0.0001 \\
17 & 0.0000 \\
18 & 0.0000 \\
19 & 0.0000 \\
20 & 0.0000 \\
\hline
\end{tabular}

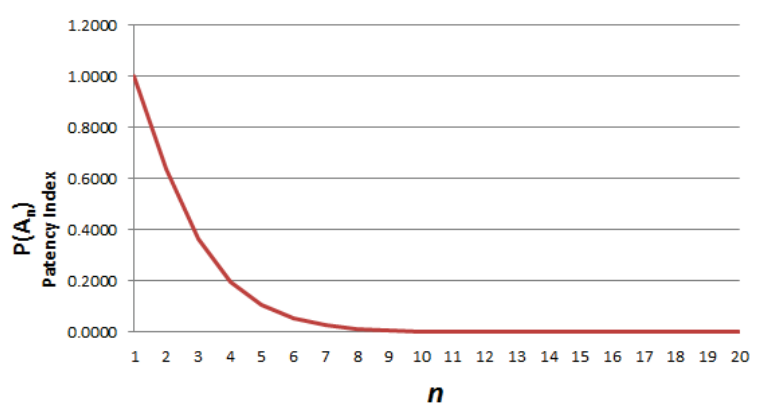

Figure 2. Plot of $P\left(A_{n}\right)$ (along the ordinate) vs. $n$ (along the abscissa) for Linear Acenes $A_{1}-A_{20}$.

$$
P\left(A_{n}\right)=\frac{f(n)}{{ }^{5 n+1} C_{n}}
$$

then sufficient accuracy for $P\left(A_{n}\right)$ is obtainable simply by using

$$
\frac{(3+\sqrt{8})^{n+1}}{\sqrt{32}}
$$

for $f(n)$, without the need for the second (subtractive) term in equation (5), or for the process of extracting the 'integral-part-of', as is done in equation (6), above. Table 1 shows (correct to 4 decimal places) values of $A_{n}$, so calculated, for several $n$ in the range $1 \leq n \leq 20$. It will be seen from the Table that, by about $n=17, P\left(A_{n}\right)$ is effectively zero. (In a chemical context, however, this is in fact irrelevant because stable Linear Acenes do not exist beyond $n=$ 5 (Pentacene) ${ }^{[21,22]}$.) It will be observed that $P\left(A_{n}\right)$ is roughly halved as one goes along the series - that is, each time $n$ is increased by 1 . Between $n=1$ and about $n=15, P\left(A_{n}\right)$ thus can be said approximately to exhibit an exponential decay, as is illustrated in Figure 2.

\section{EXTENSION TO OTHER HOMOLOGOUS BENZENOID SERIES}

Such an exponential decrease is not observed in the case of some physical or chemical properties, ${ }^{[23]}$ such as melting points and boiling points, in the first five (chemically stable) members of the Linear-Acene series, $A_{n}$. (Inverse) correlation with such properties might possibly have been hoped for if the indices of Patency and Spanning-Tree Density are in some way quantifying 'molecular complexity'. However, this lack of correlation in the Linear Acene series just referred to is, perhaps, only to be expected when it is realised that successive annellation to form the Linear-Acene series $\left(A_{n}\right)$ is not the only way in which benzenoid rings may be linearly tessellated: in addition to the [n]-Acenes, there are also 

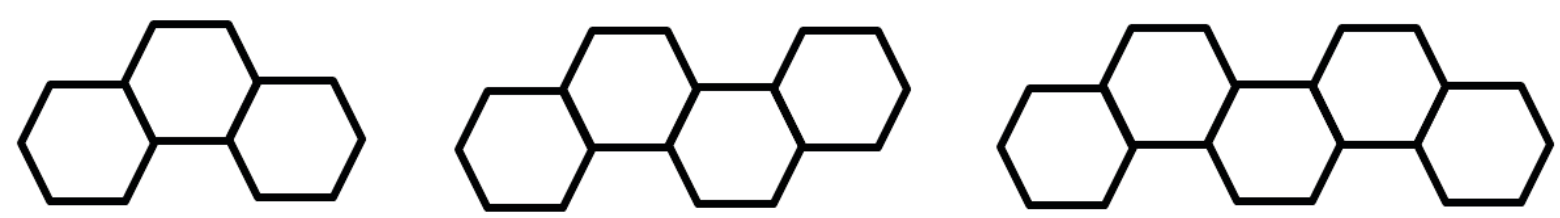

Figure 3. The molecular graphs of the first three members of the [n]-Phenacene series $\left(P h_{n}\right.$, with $n=3,4$ and 5).
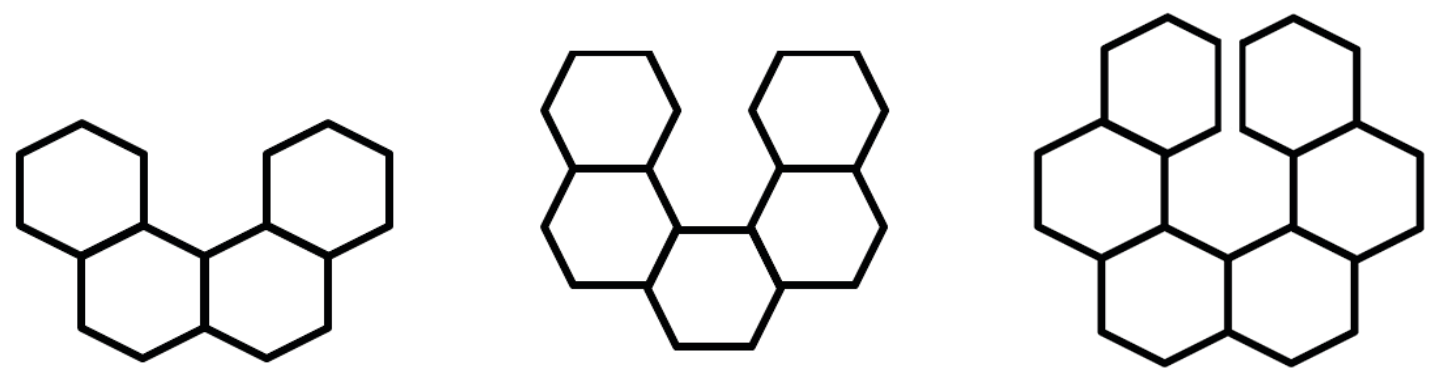

Figure 4. The molecular graphs of the first three members of the $[n]$-Helicene series $\left(H_{n}\right.$, with $n=4,5$ and 6$)$.

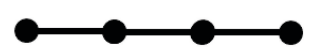

(a)

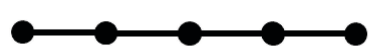

(b)

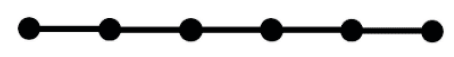

(c)

Figure 5. The inner duals $[17,29]$ of the molecular graphs (when embedded on a sphere or a plane) of (a) $A_{4}, P h_{4}$ and $H_{4}$; (b) $A_{5}$, $P h_{5}$ and $H_{5} ;$ (c) $A_{6}, P h_{6}$ and $H_{6}$.

(a) the $[n]$-Phenacene series $\left(P h_{n}\right)-n>2-$ in which successive addition of benzenoid rings is effected in a zig-zag fashion to yield the structures of Phenanthrene ([3]-Phenacene), Chrysene ([4]-Phenacene), Picene ([5]-Phenacene), etc., as in Figure 3, and

(b) the $[n]$-Helicene series $\left(H_{n}\right)-n>3-$ in which rings overlap physically in space and, helped also by their attached protons, crowd each other out so that the molecule itself is geometrically (though not graphtheoretically) non-planar. ${ }^{[24-28]}$ This results in structures such as [4]-Helicene (also known as 3,4 Benzophenanthrene and Tetrahelicene), [5]-Helicene (Pentahelicene), [6]-Helicene (Hexahelicene), etc. Some of these are illustrated in Figure 4.

However, the graphs that are the inner-duals ${ }^{\dagger},[17,29]$ arising in the spanning-tree counting theorem of Gutman et al. [17] for the molecular graphs of the series $A_{n}, P h_{n}$, and $H_{n}$ (when embedded on a sphere or a plane) are in fact the same (for the identical values of $n$ ) - these inner duals are shown in Figure 5 for $n=4,5$ and $6-$ and, in addition, because the number of edges and vertices in the molecular graphs $A_{n}, P h_{n}$ and $H_{n}$ are likewise all the same (for a given value of $n$ ), it thus follows that (again, for a specified value of $n$ )

$$
\operatorname{STD}\left(A_{n}\right)=\operatorname{STD}\left(P h_{n}\right)=\operatorname{STD}\left(H_{n}\right) \text {. }
$$

Furthermore, because of the result presented in Ref. [2] ensuring that $P(G)=\operatorname{STD}(G)$ for (graph-theoretically) planar $G$ in a planar embedding, and, in addition, because the number of edges and faces (rings) in the molecular graphs $A_{n}$, $P h_{n}$ and $H_{n}$ are again all the same (for a given value of $n$ ), it thus also follows that (once more, for a specified value of $n$ ):

$$
P\left(A_{n}\right)=P\left(P h_{n}\right)=P\left(H_{n}\right)
$$

\footnotetext{
${ }^{\dagger}$ It should be noted in passing that although (for a common value of $n$ ) the graphs that are the inner duals of the three series $A_{n}, P h_{n}$ and $H_{n}$ (when the molecular graphs of these homologous series are embedded on a sphere or a plane) are identical, what Balaban \& Harary ${ }^{[30]}$ called the 'characteristic graphs' of $A_{n}, P h_{n}$ and $H_{n}$ are different. This is because ${ }^{[17]}$ the so called 'characteristic graphs' of benzenoid systems, as defined by Balaban \& Harary in Ref. [30], are in fact not actually graphs at all, because they contain geometrical information, in addition to information about the connectivity of the benzenoid rings - which is solely what is conveyed by the inner duals of the molecular graphs of these three series, $A_{n}, P h_{n}$ and $H_{n}$ (illustrated in Figure 5 for $n=4,5$ and 6 ).
} 

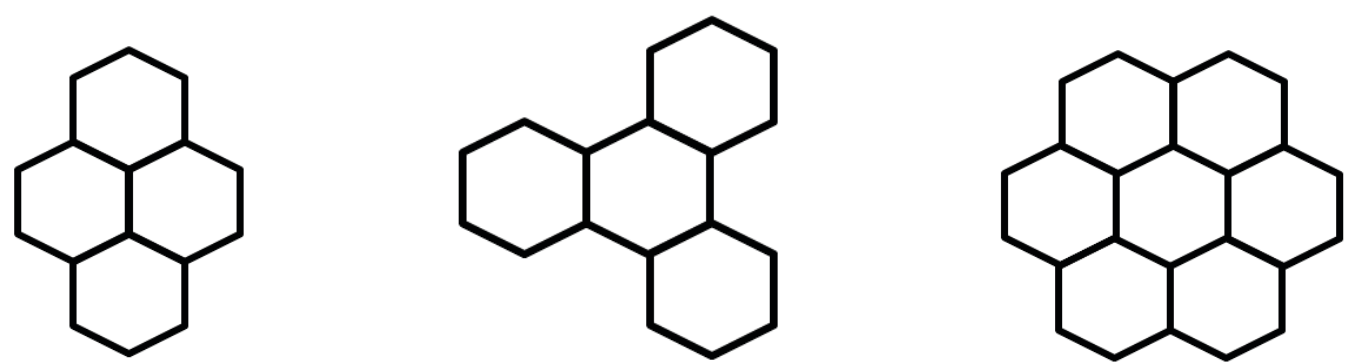

Figure 6. The molecular graphs of Pyrene (left), Triphenylene (middle) and Coronene (right).

\section{APPLICATION TO OTHER TYPES OF CONDENSED BENZENOID SYSTEMS}

Because of the identity of the inner duals ${ }^{[17,29]}$ for corresponding members of the three series $A_{n}, P h_{n}$, and $H_{n}$ (that is, members of these series for a specified value of $n$ ), and because, furthermore, analogous members of $A_{n}, P h_{n}$, and $H_{n}$ contain exactly the same number of edges, vertices and rings, the Patency Index ${ }^{[2]}$ and the Index of Spanning-Tree Density ${ }^{[1]}$ are not able to distinguish between corresponding members of the three series. This is clearly a shortcoming and it is possible that the molecular graphs of non-linear benzenoids, variously condensed such as Pyrene, Triphenylene and Coronene (Figure 6) would be more fruitful benzenoid systems for exploring the utility of the novel index $P(G)$ and the longer-established one, $\operatorname{STD}(G)$, though it would be more difficult to obtain results for them in closed form.

The three homologues series $A_{n}, P h_{n}$, and $H_{n}$ with identical inner duals ${ }^{[17]}$ (see Figure 5), when the molecular graphs of the series are embedded on a sphere or in a plane - do, though, have the advantage that, as shown in this note, their indices whose reciprocals are claimed to reflect the intricacy ${ }^{[1,2]}$ of the individual members of these three series - namely, STD $(G)$ and $P(G)$ - may be expressed in closed form whereas, for more randomly condensed systems such as the four-ringed benzenoids Pyrene and Triphenylene (left-hand and middle of Figure 6, respectively) or even symmetrically condensed systems such as the seven-ringed Coronene (right-hand of Figure 6), each structure would have to be considered individually. For example, for the above three systems,

$$
\text { /(Pyrene) }=\operatorname{det}\left(\begin{array}{cccc}
6 & -1 & 0 & -1 \\
-1 & 6 & -1 & -1 \\
0 & -1 & 6 & -1 \\
-1 & -1 & -1 & 6
\end{array}\right)=1092
$$

so that

$$
P(\text { Pyrene })=\frac{1092}{{ }^{19} C_{4}} \approx 0.2817,
$$

and

$$
\text { /(Triphenylene) }=\operatorname{det}\left(\begin{array}{cccc}
6 & -1 & -1 & -1 \\
-1 & 6 & 0 & 0 \\
-1 & 0 & 6 & 0 \\
-1 & 0 & 0 & 6
\end{array}\right)=1188
$$

so that

$$
P(\text { Triphenylene })=\frac{1188}{{ }^{21} C_{4}} \approx 0.1985,
$$

indicating, according to the criteria established in Refs. [1] $\&$ [2], that the less-condensed Tripheylene is more intricate than the more-condensed Pyrene, which likewise comprises four benzenoid rings. Furthermore:

/(Coronene) = det $\left(\begin{array}{ccccccc}6 & -1 & 0 & 0 & 0 & -1 & -1 \\ -1 & 6 & -1 & 0 & 0 & 0 & -1 \\ 0 & -1 & 6 & -1 & 0 & 0 & -1 \\ 0 & 0 & -1 & 6 & -1 & 0 & -1 \\ 0 & 0 & 0 & -1 & 6 & -1 & -1 \\ -1 & 0 & 0 & 0 & -1 & 6 & -1 \\ -1 & -1 & -1 & -1 & -1 & -1 & 6\end{array}\right)=176400$,

so that

$$
P(\text { Coronene })=\frac{176400}{{ }^{30} C_{7}} \approx 0.0866,
$$

indicating that, of the three structures in Figure 6, symmetrical, seven-ringed Coronene has the greatest intricacy.

\section{CONCLUDING REMARKS}

As already pointed out, the advantage of considering any or all of the three homologous series studied here is that, because both their Spanning-Tree Densities and Patency Indices may be expressed in closed form, we have been able to 
show that both indices tend to zero (indicating increasing intricacy) as the number of benzenoid rings in each of the three series becomes infinitely large. It is clear that, for a given $n$, the value of both the Patency Index and of the Index of Spanning-Tree Density is the same for $A_{n}, P h_{n}$ and $H_{n}$. It thus follows that the value of neither index is likely to be directly related to some physical or chemical datum for those series of compounds. This, however, does not preclude the possibility that, within each series, either index may exhibit correlation with some such datum. This is a matter for practical investigation.

\section{REFERENCES}

[1] R. B. Mallion, N. Trinajstić, MATCH Commun. Math. Comput. Chem. 2003, 48, 97.

[2] E. C. Kirby, R. B. Mallion, P. Pollak, P. J. Skrzyński, Croat. Chem. Acta, 2017, 90, 75.

[3] D. Bonchev, 'The problems of computing molecular complexity' in Computational Chemical Graph Theory, (Ed D. H. Rouvray), Nova Science Publishers, New York, 1990, Chapter 2, pp. 34-63.

[4] M. M. Waldrop, Complexity, Touchstone/Simon \& Schuster, New York, 1992.

[5] B. Kaye, Chaos and Complexity, VCH, Weinheim, Germany, 1993.

[6] D. Bonchev, D. H. Rouvray, Complexity in Chemistry, Biology and Ecology, Mathematical and Computational Chemistry Series, (P. G. Mezey (Series Ed.)), Springer, New York, 2005.

[7] W. T. Tutte, Graph Theory As I Have Known It, Oxford Lecture Series in Mathematics and its Applications, 11. (Series Eds.: G. Ball, D. Welsh), Clarendon Press, Oxford, UK, 1998, especially pp. 7, 26, 27, 99 \& 112.

[8] R. J. Wilson, Introduction to Graph Theory, First Edition, Oliver \& Boyd, Edinburgh, 1972, pp. 46-48; Fifth Edition, Prentice Hall, Upper Saddle River, New Jersey, USA, 2010, p. 63.

[9] J. W. Moon, Counting Labelled Trees, Canadian Mathematical Monographs, Ottawa, 1970, Chapter 5, especially pp. 64-65.

[10] D. M. Cvetković, M. Doob, H. Sachs, Spectra of Graphs - Theory and Applications, First Edition, Deutscher Verlag der Wissenschaften, Berlin (East), 1979; Second Edition, Academic Press, New York, 1980; Third Edition, Johann Ambrosius Barth, Heidelberg \& Leipzig, 1995, especially pp. 38-41 and p. 220.
[11] H. Fleischner, Eulerian Graphs and Related Topics Part 1, Volume 2, North Holland, Amsterdam, 1991, pp. IX 73-IX 78.

[12] T. K. Dickens, R. B. Mallion, Croat. Chem. Acta 2013, 86, 387.

[13] T. K. Dickens, R. B. Mallion, MATCH Commun. Math. Comput. Chem. 2016, 76, 297.

[14] E. C. Kirby, R. B. Mallion, P. Pollak, P. J. Skrzyński, Croat. Chem. Acta 2016, 89, 403.

[15] E. C. Kirby, D. J. Klein, R. B. Mallion, P. Pollak, H. Sachs, Croat. Chem. Acta 2004, 77, 263.

[16] E. C. Kirby, R. B. Mallion, P. Pollak, 'Counting spanning trees in toroidal fullerenes' in The Mathematics and Topology of Fullerenes, (Series Eds.: F. Cataldo, P. Milani), (Volume Eds.: F. Cataldo, A. Graovac, O. Ori ), (Foreword: Sir Harold Kroto), Carbon Materials: Chemistry and Physics Volume 4: Springer, Dordrecht, Heidelberg, London \& New York, 2010, Chapter 10, pp. 187-204.

[17] I. Gutman, R. B. Mallion, J. W. Essam, Mol. Phys. 1983, 50, 859.

[18] S. J. Cyvin, I. Gutman, Kekulé Structures in Benzenoid Hydrocarbons, Lecture Notes on Chemistry Series, Springer-Verlag, Berlin (West), 1988.

[19] S. J. Cyvin, I. Gutman, Introduction to the Theory of Benzenoid Hydrocarbons, Springer-Verlag, Berlin (West), 1989.

[20] D. Janežić, A. Miličević, S. Nikolić, N. Trinajstić, Graph Theoretical Matrices in Chemistry (Eds.: I. Gutman, B. Furtula), Mathematical Chemistry Monographs, Faculty of Science, University of Kragujevac, Serbia, 2007, Chapter 2, pp. 5-50, especially pp. 46-49.

[21] J. E. Anthony, Angewandte Chem. Int. Edn. 2008, 47, 452.

[22] S. S. Zade, M. Bendikov, Angewandte Chem. Int. Edn. 2010, 49, 4012.

[23] W. M. Hayes (Editor-in-Chief) CRC Handbook of Chemistry and Physics, 97 th Edition, 2016-2017, CRC Press, Boca Raton, Florida, USA, 2016.

[24] M. S. Newman, D. Lednicer, J. Am. Chem. Soc. 1956, $78,4765$.

[25] C. A. Coulson, C. W. Haigh, Tetrahedron 1963, 19, 527.

[26] R. H. Martin, Tetrahedron 1964, 20, 897.

[27] C. W. Haigh, R. B. Mallion, Mol. Phys. 1971, 22, 945.

[28] R. H. Martin, Angewandte Chem. Int. Edn. 1974, 13, 649.

[29] D. M. Cvetković, I. Gutman, Publ. Inst. Math. (Beograd) 1981, 29, 49.

[30] A. T. Balaban, F. Harary, Tetrahedron 1968, 24, 2505. 